THE ASTROPHYSICAL JouRNAL, 409:770-775, 1993 June 1

(C) 1993. The American Astronomical Society. All rights reserved. Printed in U.S.A.

\title{
A NEW LUMINOUS BLUE VARIABLE: R143 IN 30 DORADUS
}

\author{
Joel WM. PARKer ${ }^{1,2,3}$ AND Geoffrey C. Clayton ${ }^{2,4}$ \\ Center for Astrophysics and Space Astronomy, Box 389, University of Colorado, Boulder, Colorado 80309-0389 \\ Cláudia Winge ${ }^{1}$ \\ Departamento de Astronomia, Instituto de Física, Universidade Federal do Rio Grande do Sul, \\ Av. Bento Gonçalves, 9500-C.P. 15051, CEP 91500 Porto Alegre, RS, Brasil \\ AND \\ Peter S. Conti ${ }^{5}$ \\ Joint Institute for Laboratory Astrophysics, Box 440, University of Colorado, Boulder, Colorado 80309-0440 \\ Received 1992 August 10; accepted 1992 November 23
}

\begin{abstract}
We have discovered that R143 in the Large Magellanic Cloud is a luminous blue variable (LBV), the first and perhaps the lone LBV in the central cluster of 30 Doradus, and only the sixth known LMC LBV. Photometric and spectroscopic observations over the past $40 \mathrm{yr}$ indicate that during that time R143 moved redward (changing from an F5 to F8 supergiant), then blueward (possibly becoming as early as O9.5), and is now moving back to the red (currently appearing as a late B supergiant). Similarly, the $V$ magnitude of the star has changed by at least 1.4 mag. Images of R143 show very unusual filaments of nebulosity extending from the star to a shell at a distance of $3.5 \mathrm{pc}$, perhaps due to a similar ejection mechanism that created the spiral jets and shell associated with AG Car, another LBV.
\end{abstract}

Subject headings: ISM: individual (30 Doradus) — stars: variables: other (luminous blue variables) supergiants

\section{INTRODUCTION}

Luminous blue variables are hot and massive stars that show strong photometric and spectroscopic variations; they include P Cygni stars, S Doradus stars, and Hubble-Sandage variables (but not Wolf-Rayet stars). Conti (1984) first coined the term luminous blue variable (LBV), and Lamers (1986) and Humphreys (1989) give overviews of their properties which are outlined here. As their name implies, LBVs are characterized by their variability. They vary by 1-2 mag on time scales of a few decades, and less frequently-perhaps once every few centuries - they can have eruptions that increase their brightness by more than 3 mag. These stars also exhibit large temperature variations. During the quiescent phases of minimum brightness they appear to be blue B-type supergiants with temperatures $\gtrsim 20,000 \mathrm{~K}$. However, during the periods of maximum brightness they resemble late-type $(\mathrm{A}-\mathrm{F})$ supergiants with temperatures around $8000 \mathrm{~K}$; this may be due to very extended, cool, and optically thick envelopes surrounding these stars as modeled by Leitherer et al. (1985) and Davidson (1987). It has been suggested (e.g., Wolf, Appenzeller, \& Stahl 1981; Lamers 1986; Stahl \& Wolf 1986; and references therein) that the bolometric magnitudes of LBVs remain roughly constant throughout these variations.

\footnotetext{
${ }^{1}$ Visiting Astronomer, Cerro Tololo Inter-American Observatory, National Optical Astronomy Observatories, operated by the Association of Universities for Research in Astronomy, Inc., under a cooperative agreement with the National Science Foundation.

${ }^{2}$ Guest Observer with the International Ultraviolet Explorer Satellite, which is sponsored and operated by the National Aeronautics and Space Administration, the Science Research Council of the United Kingdom, and the European Space Agency.

3 Now at The Laboratory for Astronomy and Solar Physics, Mail Code 681, Goddard Spaceflight Center, National Aeronautics and Space Administration, Greenbelt, MD 20771; e-mail: joel@hrssun.gsfc.nasa.gov.

4 e-mail:gclayton@fenway.colorado.edu.

5 e-mail:pconti@jila.colorado.edu.
}

It is generally believed that LBVs are massive stars in the late stages of their evolution. As they careen across the H-R diagram in repeated blue-red-blue excursions, they lose mass through constant winds and occasional outbursts. This scenario may lead an LBV to a Wolf-Rayet phase or even a supernova, which perhaps could occur either during the outburst phase as a red supergiant or the quiescent phase as a blue supergiant such as the progenitor of SN 1987A. These temperature variations should not be confused with the "blue loops" that are thought to occur in the post-main-sequence evolution of massive stars due to nuclear evolution; in LBVs the variability is due to photospheric instabilities and changes. Only about 31 stars in the local group of galaxies are known to be LBVs (Bohannan 1989; Humphreys 1989, 1992), and five of these rare and interesting stars are in the LMC. The recent discovery of large spectroscopic and photometric variations of the star R143 in 30 Doradus has led us to suspect that it may be a member of the LBV class. New observations presented here confirm R143 as the sixth known LBV in the LMC.

$\mathrm{R} 143$ is a supergiant in the central cluster of 30 Doradus. It is located at $\alpha(2000.0)=5^{\mathrm{h}} 38^{\mathrm{m}} 51^{\mathrm{s}} .6$ and $\delta(2000.0)=$ $-69^{\circ} 08^{\prime} 06^{\prime \prime}$, about $2^{\prime} .2$ southeast of R136, which is the core cluster of 30 Dor (finding charts can be found in Feast, Thackeray, \& Wesselink 1960; Melnick 1985; and Parker 1992). R143 has never been the specific subject of any study except for a few comments by Feast (1961), but due to its proximity to the central cluster of 30 Dor, there are a few scattered observations of this star from studies of the region. Other designations for R143 are HDE 269929, 30 Dor star 15 in Westerlund (1961), 30 Dor IR 19 in McGregor \& Hyland (1981); star 1575 in Parker (1992), ${ }^{6}$ and IRS 141 in Hyland et al. (1992).

\footnotetext{
${ }^{6}$ Parker (1992) also detects the presence of a faint $(V \approx 17.5 \mathrm{mag})$ star 1.5 from R143.
} 
TABLE 1

\begin{tabular}{ccccccl}
\hline \multicolumn{7}{c}{$U B V R I$ OBSERVATIONS } \\
\hline \hline Observation Dates & $U-B$ & $B-V$ & $V$ & $V-R$ & $R-I$ & \multicolumn{1}{c}{ Source } \\
\hline $1957-1960 \ldots \ldots \ldots \ldots \ldots$ & $\ldots$ & +0.74 & 10.62 & +0.40 & +0.35 & Westerlund 1961 \\
$1981 \ldots \ldots \ldots \ldots \ldots \ldots$. & -0.59 & +0.44 & 12.03 & +0.30 & +0.29 & Clayton \& Martin 1985 \\
$1985 \ldots \ldots \ldots \ldots \ldots \ldots$. & -0.52 & +0.44 & 12.01 & $\ldots$ & $\ldots$ & Parker 1992 \\
\hline
\end{tabular}

P. Massey and K. DeGioia-Eastwood made $U B V$ CCD observations of 30 Dor in 1985 as part of a study of the stellar content of $\mathrm{OB}$ associations in the Magellanic Clouds. These images were analyzed by Parker (1992), who discovered that the photometry (Table 1) greatly differs from that of Westerlund (1961) and from the expected colors for a late F-type supergiant as R143 was classified by Feast et al. (1960). ${ }^{7}$ However, the Parker (1992) results agree extremely well with those of Clayton \& Martin (1985) obtained only four years earlier, and so we suspect that the discrepancy was due to a large intrinsic variability of R143 rather than misidentification. This is supported by the photometric changes seen when one compares the $J H K$ infrared observations (Glass 1974; McGregor \& Hyland 1981; Hyland et al. 1992) shown in Table 2. Also, Lortet (1992) reports that it was not possible to get CORAVEL radial velocity measurements of R143 from observations by H. Lindgren in 1990 and E. Maurice in 1991. CORAVEL is able to measure velocities of stars of spectral types later than about A5, indicating that R143 was an earlier type star or that many absorption lines were filled in with emission.

Observations of R143 were first published by Feast et al. (1960); they classified it as an F7 Ia, which they note is a compromise between two observations. The star appears to have changed from an F5 Ia (in 1953 October) to an F8 Ia (in 1956 September), and they state that: "It appears probable that there has been a real change of type between the epochs of these two plates" (Feast et al. 1960; dates of the observations were obtained from Lloyd Evans 1992). However, the lack of appropriate standards taken with these observations make the classifications somewhat uncertain (Lloyd Evans 1992).

There have been no spectroscopic observations and very little photometry of R143 published since then. But these sparse data led us to believe that the star had undergone large photometric and spectroscopic changes, which required new observations for verification. This paper presents new visible and ultraviolet observations confirming that R143 indeed is a LBV.

\section{NEW OBSERVATIONS}

A visible spectrum from 3650 to $7200 \AA$ with a resolution of $8 \AA$ was obtained by C. Winge in 1992 May with the "2DFRUTTI" detector on the $1 \mathrm{~m}$ telescope at Cerro Tololo InterAmerican Observatory (CTIO). Seeing was roughly 1".5. The spectrogram is shown in Figure 1. An additional spectrum centered on $\mathrm{H} \alpha$ was obtained by R. Schommer in 1992 May at CTIO on the $1.5 \mathrm{~m}$ telescope. Even though the visible spectra have been sky-subtracted, they are heavily contaminated with nebular emission, in particular: $\mathrm{H} \alpha, \mathrm{H} \beta, \mathrm{H} \gamma,[\mathrm{O}$ III] $\lambda \lambda 4959$, 5007, [O II $] \lambda 4363$, and [N II] $\lambda \lambda 6548,6583$. (This emission is probably from the 30 Dor nebula, and not the star.) Although these spectrograms were not adequate to classify R143, they

\footnotetext{
${ }^{7}$ Clayton (1983) was the first to note the discrepancies in the observational data of R143, but the variations were assumed to be due to misidentifications.
}

indicate that it is clearly an early-type star: the continuum is quite blue, and there are no indications of the metallic lines seen in later type supergiants.

We obtained ultraviolet (UV) spectra of R143 with the International Ultraviolet Explorer (IUE) on 1992 June 14 and 18. Two short-wavelength spectra (SWP 44923, 44951) and one long-wavelength spectrum (LWP 23299) were obtained at low dispersion through the large aperture. These data were reduced at the Regional Data Analysis Facility at the University of Colorado, Boulder. Absolutely calibrated spectra were produced from the line-by-line IUE data files using the Optimal IUE data extraction software (Kinney, Bohlin, \& Neill 1991). A three-point boxcar average has been applied to the data to match the resolution of the spectrograph (6 $\AA$ ). This spectrogram also is shown in Figure 1.

Since the visible spectrum was contaminated by emission lines, the UV spectrum was used for stellar classification. R143 appears to have been a B8-9 I star during these recent UV observations. Temperature and luminosity classifications were made on the basis of the $\mathrm{Mg}$ II, $\mathrm{Fe}$ II, $\mathrm{Fe}$ III, $\mathrm{C}$ II, and $\mathrm{Si}$ II lines. There is no indication of strong emission in the UV spectrum. The UV spectral features are a good match to those in the star $\mathrm{Sk}-6982$, which is an LMC standard star classified as B9 Ia by its visible spectrum (Fitzpatrick 1991). The IUE spectrum also is similar to that of the Galactic LBV P Cygni (Lamers, de Groot, \& Cassatella 1983), with numerous lines of singly and doubly ionized metals, $\mathrm{C}$ IV and Si IV absorptions, and a possible $\mathrm{P}$ Cygni profile in $\mathrm{Mg}$ II. The high-resolution spectrum of $P$ Cygni showed that these low-ionization lines are formed in a cool wind and suggest a late B spectral type. However, the energy distribution and photospheric lines in $\mathrm{P}$ Cygni indicate the underlying star is actually a B1.5 supergiant with $T_{\text {eff }}=$ $19,000 \mathrm{~K}$. The $\mathrm{C}$ IV and $\mathrm{Si}$ IV photospheric lines in R143 are much weaker than in P Cygni, but its energy distribution does suggest that the underlying star in R143 also may be earlier than B8. This is supported by comparisons of the equivalent widths of the Si III $\lambda 1300$ and Al III $\lambda 1860$ absorption lines with the Shore \& Sanduleak (1984) calibrations, which indicate an early-B spectral type.

The Ultraviolet Imaging Telescope (UIT) obtained photometry of 30 Dor stars during the Astro 1 shuttle mission (Cheng et al. 1992). We made comparisons between the UV photometry of R143 from the UIT observations (Stecher 1992) and the IUE data convolved with the transmission functions of the UIT filters (Stecher et al. 1992). The expected errors

TABLE 2

JHK OBSERVATIONS

\begin{tabular}{crccl}
\hline \hline $\begin{array}{c}\text { Observation } \\
\text { Dates }\end{array}$ & \multicolumn{1}{c}{$\boldsymbol{J}$} & $\boldsymbol{J}-\boldsymbol{K}$ & $\boldsymbol{H}-\boldsymbol{K}$ & \multicolumn{1}{c}{ Source } \\
\hline $1973 \ldots \ldots \ldots \ldots$ & 9.60 & +0.50 & +0.25 & Glass 1974 \\
$1976,1977 \ldots \ldots$ & 10.71 & +0.46 & +0.18 & McGregor \& Hyland 1981 \\
$1982 \ldots \ldots \ldots \ldots$ & 11.07 & +0.29 & $\ldots$ & Hyland et al. 1992 \\
\hline
\end{tabular}



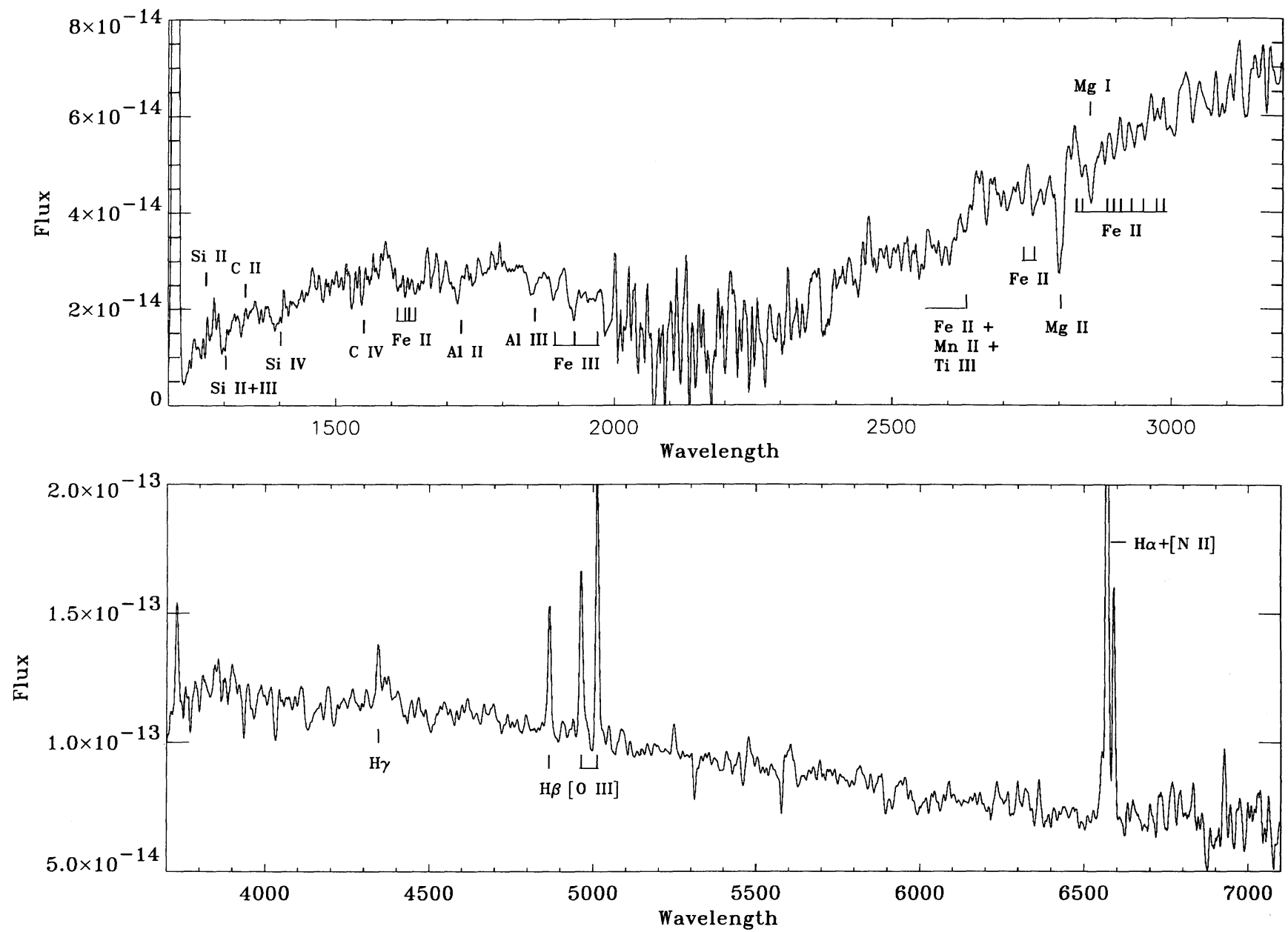

FIG. 1.-The top panel shows the ultraviolet spectrum of R143 observed with IUE. The two SWP spectrograms (images 44923 and 44951 with exposure times of 7500 and $10,800 \mathrm{~s}$, respectively) have been averaged and merged with the LWP spectrogram (image 23299 with an exposure time of $1800 \mathrm{~s}$ ) at $1984 \AA$. Line identifications are from Heck et al. (1984) and de Winter et al. (1992). The lower panel shows the optical spectrum obtained with the $1 \mathrm{~m}$ telescope at CTIO; the data were taken on a nonphotometric night, so the absolute flux variables are not reliable. The emission lines are probably from the 30 Dor nebulosity and not due to the star.

between the two observations are fairly large $(\sigma \approx 0.2 \mathrm{mag})$, but they show that the UV magnitudes of R143 have not changed by more than $2 \sigma$ between 1990 December and 1992 June.

\section{ANALYSIS}

\subsection{Reddening}

Only photometric observations exist for the period between 1956 when R143 was an F8 star and 1992 when it was a B8 star. Since this star is obviously quite variable in spectral type, and no simultaneous photometry has been obtained at any of the epochs where the spectral type is known, the reddening is uncertain. However, the photometry summarized in Tables 1 and 2 does seem to paint a consistent picture of $E(B-V)=0.65$ once variability of the spectral type is assumed. Determining the reddening of R143 is necessary for the goal of calculating the star's bolometric magnitude.

The UBVRI photometry obtained in 1981 and 1985 indicates a reddened early-type star so the reddening-free $Q$ parameter (Johnson \& Morgan 1953) can be calculated. This parameter has been calibrated for early-type supergiants using the intrinsic colors from FitzGerald (1970) and Schmidt-Kaler (1983). The 1981 and 1985 photometry in Table 1 give
$Q=-0.84$ to -0.91 , respectively, which indicates a spectral type of B1 I or possibly as early as $\mathrm{O} 9.5 \mathrm{I}$, and a reddening of $E(B-V)=0.64$ to 0.70 (assuming that LBVs have the same $U B V$ colors as normal supergiants). This spread in reddening translates to a difference of less than $0.2 \mathrm{mag}$ in the bolometric magnitude. The $1985 R$ and $I$ band data are also consistent with a $B 1$ I star and $E(B-V) \approx 0.65$ assuming a Savage \& Mathis (1979) extinction curve. (Note that the $R$ and $I$ magnitudes in Clayton \& Martin [1985] are on the Cousins system and must first be converted to the Johnson system for comparison.)

A number of studies have found a relatively large mean reddening of $\overline{E(B-V)_{30} \text { Dor }} \approx 0.4 \pm 0.2$ (e.g., Israel \& Koornneef 1979; Fitzpatrick \& Savage 1984; Lee 1990; Vacca 1991; Heap et al. 1991; Parker 1992) in the 30 Dor region compared to the overall low reddening in the LMC, supporting our value of $E(B-V)=0.65$ for R143. Parker (1992) finds $0.3 \leq E(B-V) \leq 0.7$ with a mean value of $E(B-V)=0.5$ for 15 stars within $25^{\prime \prime}$ of R143. We can also put limits on the reddening from polarization studies. Clayton, Martin, \& Thompson (1983) obtained $U B V R I$ polarimetry of R143. The best fit to the Serkowski relation found $P_{\max }=3.44 \%$ and $\lambda_{\max }=0.49 \mu \mathrm{m}$. If the polarization is all interstellar then this 
puts a lower limit on the $E(B-V)$ of about 0.4 (Serkowski, Mathewson, \& Ford 1975). However, Clayton et al. (1983) point out that the Serkowski fit to the R143 data is not very good. This combined with the known variability and supergiant/LBV nature of the star makes the presence of an intrinsic component quite likely (Serkowski 1970; SchulteLadbeck et al. 1993).

The effects of reddening seen in the UV spectrum are consistent with $E(B-V) \approx 0.65$. Determination of the reddening directly from the UV spectrum is very difficult for two reasons. First, the UV extinction curve is normally derived by comparison with an unreddened star of the same spectral type. This is hard to do for a LBV since the underlying star can have an earlier spectral type than is indicated by its UV absorption lines which are formed in the cool wind (Lamers et al. 1983). Second, the wavelength dependence of extinction toward stars in 30 Dor is made up of three components, foreground Milky Way dust $[E(B-V) \approx 0.07]$, LMC dust outside of 30 Dor, and dust local to 30 Dor (Fitzpatrick \& Savage 1984; Fitzpatrick 1985; Clayton \& Martin 1985). The dust in each of these three components has a distinct UV wavelength dependence. In particular, the far-UV extinction is steepest in the 30 Dor dust, and flattest in the Milky Way dust. The UV spectrum was dereddened using all three types of dust. The strength of the $2200 \AA$ bump is consistent with the assumed reddening and is removed well by all three wavelength dependences. For $E(B-V)=0.65$, dereddening the data with the non-30 Dor extinction curve (which appears to fit the data better than the 30 Dor or Galactic extinction curves) produces an unreddened UV energy distribution similar to a mid-type B supergiant with $T=14,000 \mathrm{~K}$. This is hotter than the B8-9 spectral type implied by the absorption lines, and consistent with the characteristics of LBVs (e.g., P Cygni as discussed in § 2).

\subsection{Spectral Types and Bolometric Magnitudes}

With the best estimate of $E(B-V)=0.65\left(A_{V}=2.0 \mathrm{mag}\right.$, for $R_{V}=3.1$ ) for $\mathrm{R} 143$, and assuming that the reddening is nonvariable, we can then use this value to interpret the photometry at other epochs and estimate the bolometric magnitudes. Spectral types as determined by photometry alone are somewhat uncertain, so the errors in the bolometric magnitudes could be on the order of $0.5 \mathrm{mag}$ due to the large change in bolometric corrections for early B-type stars.

Using $V=12.0 \mathrm{mag}$ from the 1981 and 1985 observations, a bolometric correction of -1.7 mag for a B1 I star (Humphreys \& McElroy 1984) and a LMC distance modulus of $18.5 \mathrm{mag}$ (Panagia et al. 1991), we calculate the bolometric magnitude of the star at those epochs to be $M_{\mathrm{bol}}=-10.2 \mathrm{mag}$.

The Westerlund (1961) value of $B-V$ would be consistent with $E(B-V)=0.65$ if the spectral type at that epoch were A5 I. The $1961 R$ and $I$ data are not consistent with $B-V$ for any spectral type. This could be due to the facts that these data are averages of several observations made over three years (the $B V R I$ plates were taken at different epochs), they have been converted to the Johnson system from photographic magnitudes, and there may have been contamination from nebular emission. Assuming a spectral type of A5 I, we obtain a bolometric magnitude of $M_{\mathrm{bol}}=-9.9 \mathrm{mag}$ from the data of Westerlund (1961)

The IUE data indicate that R143 was recently (1992 June) a B8-9 or earlier supergiant. A magnitude of $V=10.9$ mag was estimated from the FES image of the IUE. The resulting bolometric magnitude is $-10.8 \mathrm{mag}$ if the spectral type was B3 Ia,
$-10.3 \mathrm{mag}$ for B6 Ia, and - $10.0 \mathrm{mag}$ for B9 Ia. In comparison with the 1961 and the 1981-1985 data, these results are consistent with R143 being a mid- to late-type B supergiant with $M_{\text {bol }} \approx-10$ mag.

Infrared data are available for three epochs. Again, using $E(B-V)=0.65$ and the Savage \& Mathis (1979) extinction curve, we can estimate the spectral type and $V$ magnitude from the $J H K$ data. Using this method we find bolometric magnitudes of $-8.1 \geq M_{\text {bol }} \geq-9.3$, all systematically fainter than the values calculated using the optical and UV data. This is probably due to an infrared excess affecting the $K$-band photometry, which would make the star appear too red. Such an excess due to circumstellar shells has been seen in other LBVs (McGregor 1989). With these caveats in mind, we find the best estimate for the bolometric magnitude of R143 is -10 mag.

Figure 2 shows how the star has moved in the H-R diagram over the past $40 \mathrm{yr}$ under the assumption that the bolometric magnitude was roughly constant at $M_{\text {bol }}=-10$ mag (using visible data only) as it varied between F8 I and B1 I. From the infrared data, it appears that R143 was on the move sometime in the mid-1970s, where the $J$ magnitude increased by $1.1 \mathrm{mag}$ between 1973 and 1977 . This is supported by measurements made from the 1976 ESO sky survey plates where $B=12.0$ mag, compared to $B=11.4 \mathrm{mag}$ in 1959 and $B=12.5 \mathrm{mag}$ in 1985. However, the infrared data indicate that the color of the star did not change during this period when the magnitude was decreasing. This is probably due to contamination by radiation from the nebula.

\subsection{Faint Filaments and a Surrounding Shell}

Feast (1961) notes that R143 has " at least four, and possibly more, curved filaments of nebulosity starting on or near the star and ending either on knots of nebulosity or faint stars." These filaments are visible in the $1985 \mathrm{CCD}$ images, and the $120 \mathrm{~s}$ exposure $V$ image of the field is reproduced in Figure 3 (Plate 13). The nebulosity is visible also in the $120 \mathrm{~s}$ exposure $B$

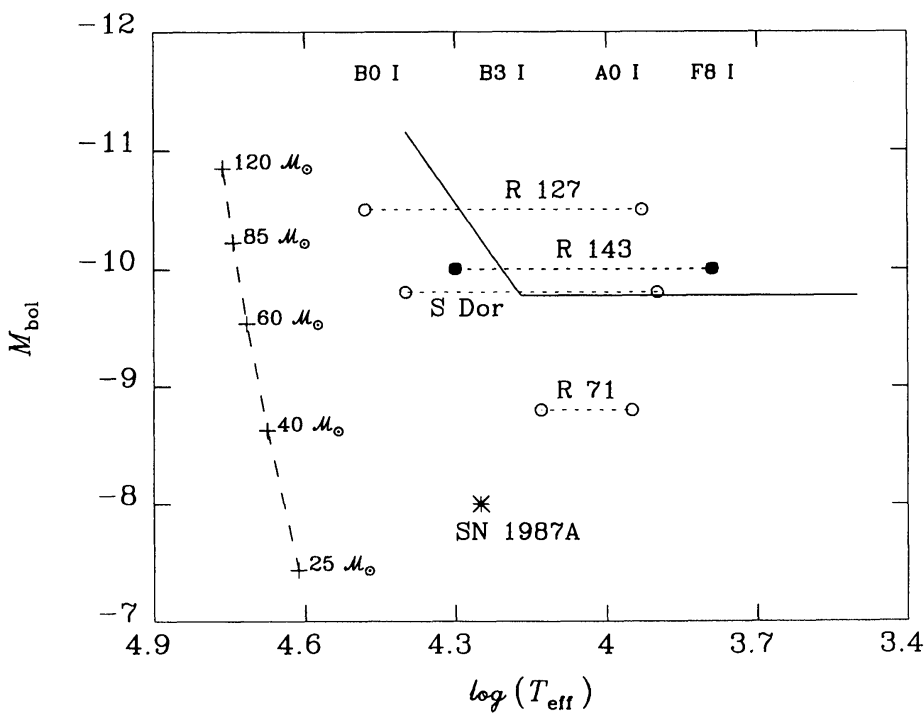

FIG. 2.-This figure shows the calculated locations of R143 on the H-R diagram (filled circles). For comparison, the open circles and the asterisk show the locations of three other LBVs in the LMC and the progenitor to SN 1987A (Humphreys 1989). The effective temperatures for supergiants are from the calibration of Humphreys \& McElroy (1984). The dashed line is the ZAMS from Maeder (1990) with $Z=0.005$. The solid lines shows the location of the luminosity limit defined by Humphreys \& Davidson (1979). 


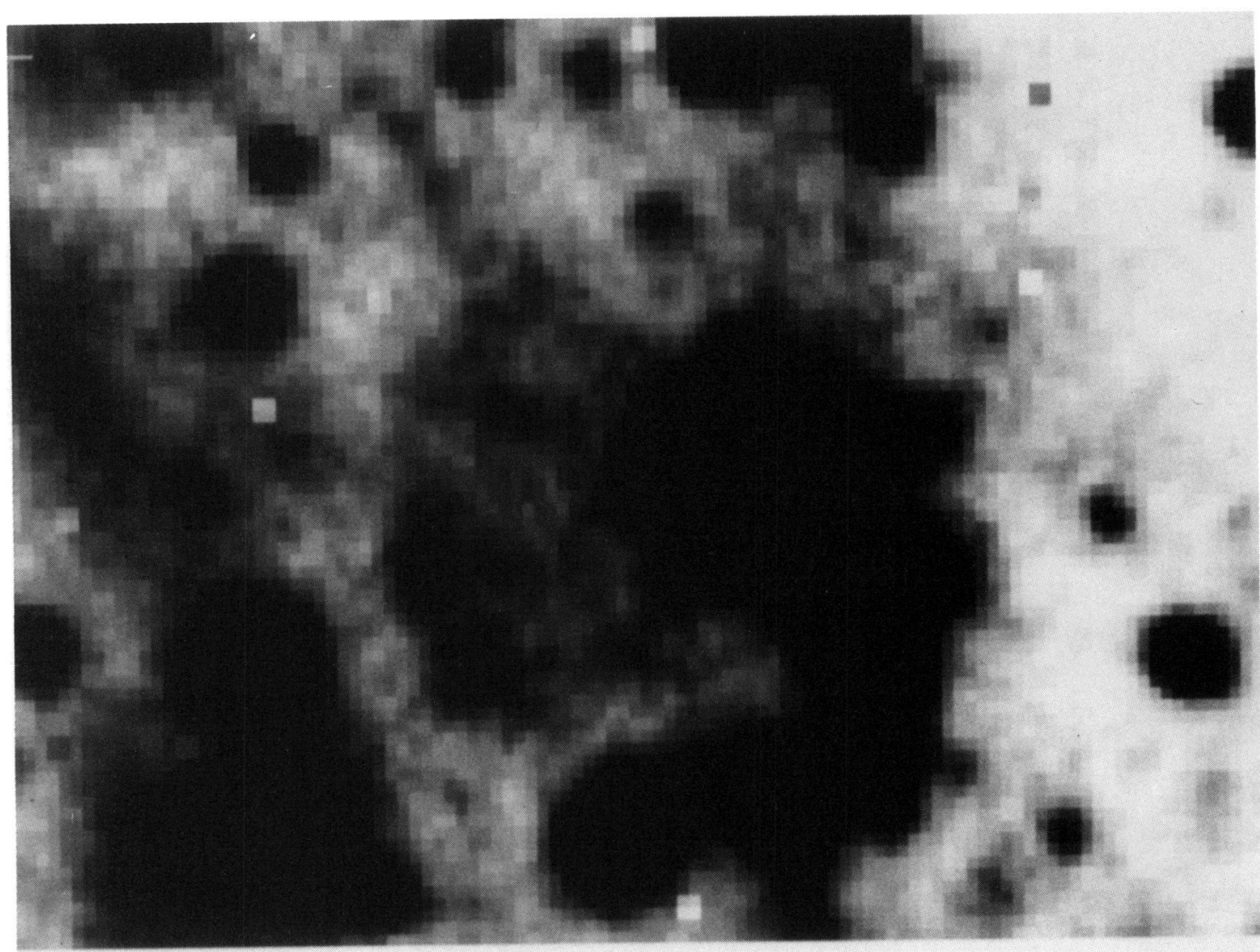

๙ัฒ

总

运

ह

m

艾

总

矛

每等

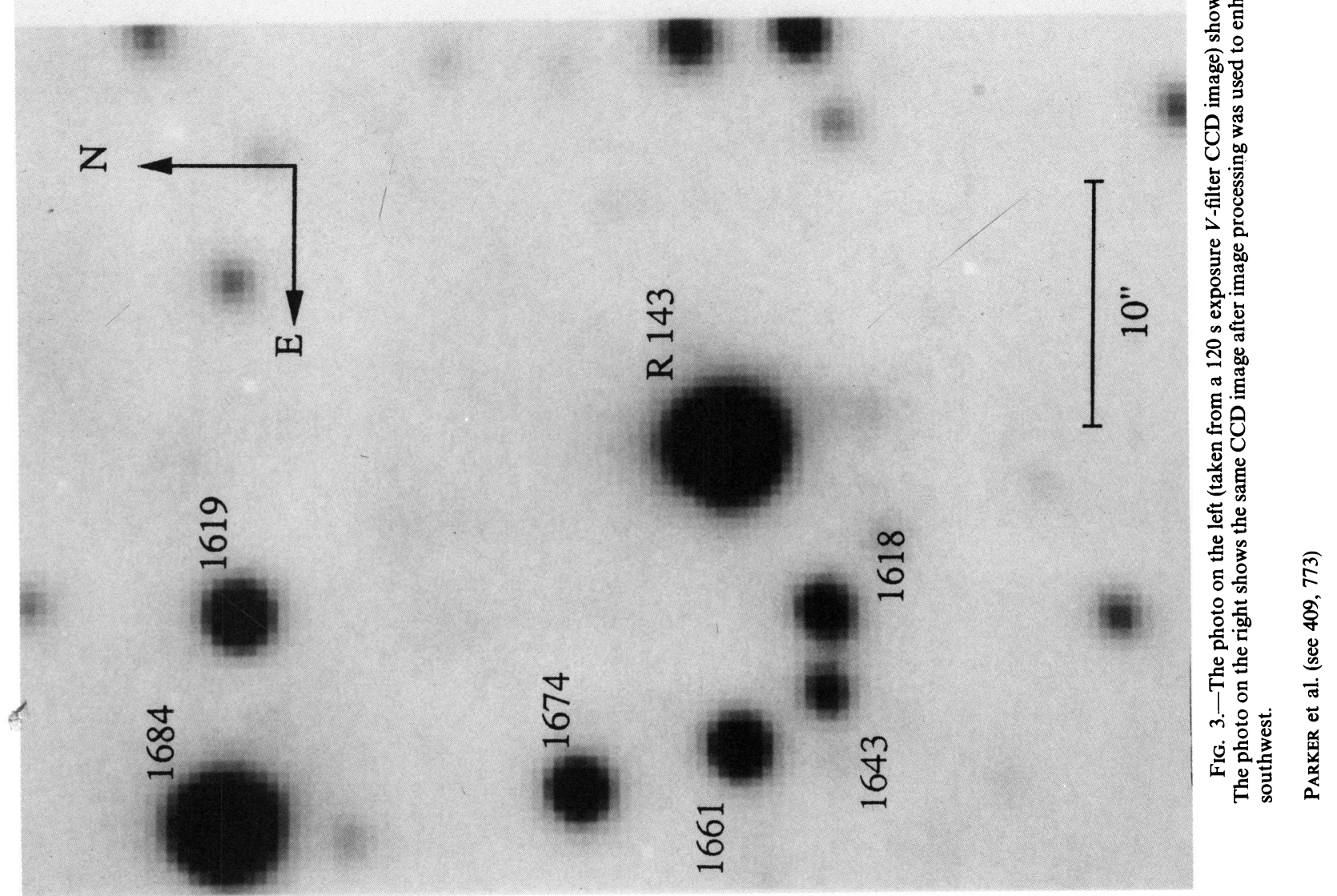


frame, but is not visible in the $800 \mathrm{~s}$ exposure $U$ frame. The filaments are about $15^{\prime \prime}$ long, which translates to a physical length of $3.5 \mathrm{pc}$. The two northeastern fingers are remarkably similar, having the same curvature, and each ending in a bright nebular knot. Likewise, the two northwestern fingers are more alike to each other than to the other filaments; they are straighter than the two northeastern fingers and do not end in such bright knots. The endpoints of these filaments trace out part of a shell which also can be seen to the southwest of R143, giving the appearance of a spoked wheel with a radius of $15^{\prime \prime}$. The shell does not seem to continue around to the east of the star, where a groúp of four stars either obscure the shell or perhaps have physically disrupted it. The apparent infrared excess discussed in $\S 3.2$ could be due to this shell (Allen 1973; McGregor 1989).

Other LBVs are known to have outbursts forming shell-like structures (see discussions in Davidson, Moffat, \& Lamers 1989 and references therein). In addition, images of AG Car using an occulting disk clearly show nebular filaments that appear to be two jets twisting around each other in a helix (Nota \& Paresce 1989). Assuming a distance to AG Car of 6 kpc (Humphreys et al. 1989), we see that the linear length of these filaments is less than $0.2 \mathrm{pc}$, considerably shorter (and perhaps indicating the filaments are younger) than those of R143. These filaments are unusual in their asymmetry: in R143 all four come from the same side of the star like the fingers from a hand. There is no indication of matching filaments/jets on the opposite side. However, the fact that AG Car also exhibits this unbalanced "twin-jet" phenomenon may point to a physical mechanism intrinsic to the stars.

\section{CONCLUSION}

The photometric and spectroscopic variations discussed here show that R143 now can be added to the list of known LBVs. It has varied from a late F-type to an early B-type supergiant, with magnitude changes of $\Delta V=1.4$ mag. It was first observed to be moving redward in the 1950 s, then blueward in the 1970s, and now has begun to move redward again from B1 to mid- or late-B. We have classified R143 in its present (1992 June) state as B8-9 Ia from its UV absorptionline spectrum, but these lines may originate in the cooler wind. The UV energy distribution indicates that the underlying star is a somewhat hotter mid-B supergiant with a reddening of $E(B-V)=0.65$ and an extinction of $A_{V}=2.0$. This relatively high reddening would explain why R143 does not appear above the luminosity limit in Humphreys \& Davidson (1979) and subsequent studies, which probably assumed smaller reddenings. These results raise the suspicion that there could be more LBVs yet to be discovered in other young stellar groups. Although LBVs are rare enough that R143 may be the lone LBV in the resolved central cluster of 30 Doradus, this can be confirmed only by more observations and searches through archives to detect long-term variability. Also, more LBVs may reside in the compact core which only now is being resolved for photometric study of its individual components (Heap, Ebbets, \& Malumuth 1993; Malumuth \& Heap 1993).

Observations of R143 over the past $40 \mathrm{yr}$ have been sparse, and the photometric observations have not been contemporaneous with the spectroscopic observations, so it is possible that R143 has varied with higher frequency and/or greater amplitude than is shown here. (An analysis of historical photographic plates of the 30 Dor region would provide a longer baseline and would give us details about the behavior of R143 in the past.) The data are consistent with the star evolving at a constant bolometric magnitude of $M_{\mathrm{bol}} \approx-10 \mathrm{mag}$. When this luminosity and the spectral type of B1 I suggested during the visual minimum quiescent phase are compared with evolutionary tracks of massive stars with $Z=0.005$ (Maeder 1990), the original mass of $\mathrm{R} 143$ was $\sim 60 \mathscr{M}_{\odot}$. The current mass is highly uncertain due to the outbursts which produced the filaments, but it must be between about $56 \mathscr{M}_{\odot}$ (the mass at the TAMS) and $46 \mathscr{M}_{\odot}$ (the mass where a $60 \mathscr{M}_{\odot}$ star turns into a Wolf-Rayet star), with corresponding ages of 3.6 and 4.0 Myr. This would imply that R143 is a member of the Scorpius OB1 phase (Walborn 1984, 1991), which is evidence of a burst of star formation earlier than the present starburst (Carina Nebula phase) centered on R136. Discussions of sequential star formation in 30 Dor can be found in McGregor \& Hyland (1981), Lortet \& Testor (1991), and Walborn \& Parker (1992).

More consistent monitoring of R143 is needed to study the current nature of its variability, to investigate the origin and structure of the filaments and shell, and to determine more precisely its physical parameters and how it fits in the LBV model. Since it is the only known LBV in the central cluster of 30 Dor, it represents an important feature in the evolutionary history of this starburst region.

We gratefully thank M. Feast and B. Westerlund for providing details of their observations, E. Fitzpatrick and M. Hanson for their help in the analysis of the $I U E$ data, R. Schommer at CTIO for obtaining spectra of R143 during his observing run, T. Stecher for supplying the UIT photometry of R143, and Y. Kondo for giving us discretionary observing time so promptly (1 week after our request). Useful discussions and feedback were provided by R. Humphreys, H. J. G. L. M. Lamers, M.-C. Lortet, S. Shore, N. R. Walborn, and an anonymous referee. This research has made use of the SIMBAD data base, operated at CDS, Strasbourg, France. Support for this study has come from NASA grant NAGW-2338, NASA Graduate Student Research Fellowship NGT-50483, a Graduate Fellowship from the University of Colorado, and a Fellowship from the Boettcher Foundation. C. Winge thanks the Brazilian institution CNPq for a Fellowship.

\section{REFERENCES}

Allen, D. A. 1973, MNRAS, 161, 145

Bohannan, B. 1989, in Physics of Luminous Blue Variables, ed. K. Davidson,

A. F. J. Moffat, \& H. J. G. L. M. Lamers (Dordrecht: Kluwer), 35

Cheng, K.-P., et al. 1992, ApJ, 395, L29

Clayton, G. C. 1983, Ph.D. thesis, Univ. Toronto

Clayton, G. C., \& Martin, P. G. 1985, ApJ, 288, 558

Clayton, G. C., Martin, P. G., \& Thompson, I. 1983, ApJ, 265, 194

Conti, P. S. 1984, in IAU Symp. 105, Observational Tests of the Stellar Evolution Theory, ed. A. Maeder \& A. Renzini (Dordrecht: Reidel), 233

Davidson, K. 1987, ApJ, 317, 760
Davidson, K., Moffat, A. F. J., \& Lamers, H. J. G. L. M., eds. 1989, Physics of Luminous Blue Variables (Dordrecht: Kluwer)

de Winter, D., Perez, M. R., Hu, J. Y., \& Thé, P. S. 1992, A\&A, 257, 632

Feast, M. W. 1961, MNRAS, 122, 1

Feast, M. W., Thackeray, A. D., \& Wesselink, A. J. 1960, MNRAS, 121, 337

FitzGerald, M. P. 1970, A\&A, 4, 234

Fitzpatrick, E. L. 1985, ApJ, 299, 219

.1991, PASP, 103,1123

Fitzpatrick, E. L., \& Savage, B. D. 1984, ApJ, 279, 578

Glass, I. S. 1974, MNRAS, 168, 249 
Heap, S. R., et al. 1991, ApJ, 377, L29

Heap, S. R., Ebbets, D., \& Malumuth, E. M. 1993, in preparation

Heck, A., Egret, D., Jaschek, M., \& Jaschek, C. 1984, IUE Low-dispersion Spectra Reference Atlas (ESA SP-1052)

InI Humphreys, R. M. 1989, in Physics of Luminous Blue Variables, ed. K. DavidII. son, A. F. J. Moffat, \& H. J. G. L. M. Lamers (Dordrecht: Kluwer), 3

.1992, private communication

Humphreys, R. M., \& Davidson, K. 1979, ApJ, 232, 409

Humphreys, R. M., \& McElroy, D. B. 1984, ApJ, 284, 565

Humphreys, R. M., Lamers, H. J. G. L. M., Hoekzema, N., \& Cassatella, A. 1989, A\&A, 218, L17

Hyland, A. R., Straw, S., Jones, T. J., \& Gatley, I. 1992, MNRAS, 257, 391

Israel, F. P., \& Koornneef, J. 1979, ApJ, 230, 390

Johnson, H. L., \& Morgan, W. W. 1953, ApJ, 117, 313

Kinney, A. L., Bohlin, R. C., \& Neill, J. D. 1991, PASP, 103, 694

Lamers, H. J. G. L. M. 1986, in IAU Symp. 116, Luminous Stars and Associations in Galaxies, ed. C. W. H. de Loore, A. J. Willis, \& P. Laskarides (Dordrecht: Reidel), 157

Lamers, H. J. G. L. M., de Groot, M., \& Cassatella, A. 1983, A\&A, 128, 299

Lamers, H. J. G. L. M., Hoekzema, N., Trams, N. R., Cassatella, A., \& Barylak, M. 1989, in Physics of Luminous Blue Variables, ed. K. Davidson, A. F. J. Moffat, \& H. J. G. L. M. Lamers (Dordrecht: Kluwer), 271

Lee, M. G. 1990, Ph.D. thesis, Univ. Washington

Leitherer, C., Appenzeller, I., Klare, G., Lamers, H. J. G. L. M., Stahl, O., Waters, L. B. F. M., \& Wolf, B. 1985, A\&A, 153, 168

Lloyd Evans, T. 1992, private communication

Lortet, M.-C. 1992, private communication

Lortet, M.-C., \& Testor, G. 1991, A\&AS, 89, 185

Maeder, A. 1990, A\&AS, 84, 139

Malumuth, E. M., \& Heap, S. R. 1993, in preparation

McGregor, P. J. 1989, in Physics of Luminous Blue Variables, ed. K. Davidson, A. F. J. Moffat, \& H. J. G. L. M. Lamers (Dordrecht: Kluwer), 165
McGregor, P. J., \& Hyland, A. R. 1981, ApJ, 250, 116

Melnick, J. 1985, A\&A, 153, 235

Nota, A., \& Paresce, F. 1989, in Physics of Luminous Blue Variables, ed K. Davidson, A. F. J. Moffat, \& H. J. G. L. M. Lamers (Dordrecht: Kluwer) 159

Panagia, N., Gilmozzi, R., Macchetto, F., Adorf, H.-M., \& Kirshner, R. P. 1991, ApJ, 380, L23

Parker, J. Wm. 1992, Ph.D. thesis, Univ. Colorado

Savage, B. D., \& Mathis, J. S. 1979, ARA\&A, 17, 73

Schmidt-Kaler, Th. 1983, in Landolt-Bernstein, New Series, Group 6, Vol. 2, Part B

Schulte-Ladbeck, R. E., Leitherer, C., Clayton, G. C., Robert, C., Meade, M. R., Drissen, L., Nota, A., \& Schmutz, W. 1993, ApJ, 407, 723 Serkowski, K. 1970, ApJ, 160, 1083

Serkowski, K., Mathewson, D. S., \& Ford, V. L. 1975, ApJ, 196, 261

Shore, S. N., \& Sanduleak, N. 1984, ApJS, 55, 1

Stahl, O., \& Wolf, B. 1986, A\&A, 154, 243

Stecher, T. P. 1992, private communication

Stecher, T. P., et al. 1992, ApJ, 395, L1

Vacca, W. D. 1991, Ph.D. thesis, Univ. Colorado

Walborn, N. R. 1984, in IAU Symp. 108, Structure and Evolution of the Magellanic Clouds, ed. S. van den Bergh \& K. S. de Boer (Dordrecht: Reidel), 243

Walborn, N. R. 1991, in Massive Stars in Starbursts, ed. C. Leitherer, N. R. Walborn, T. M. Heckman, \& C. A. Norman (STScI Symp. Ser. 5) (Cambridge: Cambridge Univ. Press), 145

Walborn, N. R., \& Parker, J. W. 1992, ApJ, 399, L87

Westerlund, B.E. 1961, Uppsala Astron Obs. Annals, 5,

Wolf, B., Appenzeller, I., \& Stahl, O. 1981, A\&A, 103, 94 